\section{Mortality in children with sickle cell disease in main- land France from 2000 to 2015}

Sickle cell disease (SCD) is the most frequent genetic disease detected by newborn screening (NBS) in France and its prevalence is currently increasing. In the last decades in high-income countries, there has been a spectacular drop in the mortality rate of SCD in childhood, from 1.1 to less than $0.15 / 100$ person-year (PY). ${ }^{1-5}$ This progress has been achieved following the implementation of preventive, supportive and therapeutic measures including early diagnosis by NBS, prevention of pneumococcal infections by antibiotic prophylaxis and vaccination, screening of stroke risk, disease-modifying or curative therapies such as hydroxycarbamide, transfusion programs, hematopoietic stem cell transplant (HSCT) and more recently gene therapy. ${ }^{6}$ Given the current growing possibilities of such treatments, there is a need to balance their respective benefit/risk ratio against real life mortality in order to deliver proper advice to parents and guide physicians. Our main objective was therefore to update mortality rates and causes of death on a nationwide scale in children with SCD during a 15-year period starting in 2000, an era of improved care. The secondary objective was to assess the impact of additional factors such as adherence or time to medical intervention upon SCD mortality.

This study was based on a national survey through the French national SCD network in order to collect all cases of death in children (age $<18$ years) diagnosed with SCD, followed-up in mainland France, with a fatal outcome recorded between January 1, 2000 and December 31, 2015. Anonymized relevant patient data were thereafter collected from medical files including time to first medical consultation after the onset of symptoms, access to medical facility ( $\geq$ or $<30$ minutes of transportation, adherence to medical appointments and/or penicillin prophylaxis, vaccination status at the time of death $){ }^{7}$ When available, autopsy reports were analyzed. A calculation of the death incidence rate in SCD children was per-

Table 1. Patient's characteristics.

\begin{tabular}{|c|c|c|c|c|}
\hline$n(\%)$ & $\begin{array}{c}\text { SCD (All genotypes) } \\
75 \text { (100) }\end{array}$ & $\begin{array}{c}\text { SS } / S \beta^{0} \text { thalassemia/S-D Punjab } \\
67(89.3)\end{array}$ & $\begin{array}{c}\text { SC/S } \beta \text { thalassemia } \\
7(9.3)\end{array}$ & $\begin{array}{l}\text { Unknown } \\
1(1.3)\end{array}$ \\
\hline Sex Ratio (M/F) & 1.3 & 1.4 & 0.7 & - \\
\hline Median age at death years (range) & $4.0(0-17.9)$ & $4.0(0-17.9)$ & $7(0.4-13.5)$ & - \\
\hline \multicolumn{5}{|l|}{ Diagnostic procedure, n (\%) } \\
\hline Newborn or prenatal screening & $70(93.3)$ & $63(94)$ & $6(85.7)$ & $1(100)$ \\
\hline Complication & $4(5.3)$ & $3(4.5)$ & $1(14.3)$ & - \\
\hline Undetermined & $1(1.3)$ & $1(1.5)$ & - & - \\
\hline \multicolumn{5}{|l|}{ Cause of death, $\mathrm{n}(\%)$} \\
\hline SCD-related & $53(70.7)$ & $49(73.1)$ & $4(57.1)$ & - \\
\hline SCD-unrelated & $18(24)$ & $14(20.9)$ & $3(42.9)$ & $1(100)$ \\
\hline Uncertain & $3(4)$ & $3(4.5)$ & - & - \\
\hline Undetermined & $1(1.3)$ & $1(1.5)$ & - & - \\
\hline \multicolumn{5}{|l|}{ Place of death, n (\%) } \\
\hline Hospital & $55(73.3)$ & $49(73.1)$ & $6(85.7)$ & - \\
\hline Home & $8(10.7)$ & $8(11.9)$ & - & - \\
\hline Abroad & $4(5.3)$ & $4(6)$ & - & - \\
\hline Undetermined & $8(10.7)$ & $6(9)$ & $1(14.3)$ & $1(100)$ \\
\hline \multicolumn{5}{|l|}{ Immunization status } \\
\hline PCV 7 or $13^{*} n(\%)$ & $44(100)$ & $38(100)$ & $6(100)$ & - \\
\hline Adequate & $38(86.4)$ & $32(84.2)$ & $6(100)$ & - \\
\hline Inadequate & $6(13.6)$ & $6(15.8)$ & - & - \\
\hline PV 23** n (\%) & $45(100)$ & $40(100)$ & $5(100)$ & - \\
\hline Adequate & $38(84.4)$ & $33(82.5)$ & $5(100)$ & - \\
\hline Inadequate & $7(15.6)$ & $7(17.5)$ & - & - \\
\hline \multicolumn{5}{|l|}{ Disease-modifying therapy***, n (\%) } \\
\hline None & - & $39(68.4)$ & - & - \\
\hline Yes & - & $18(31.6)$ & - & - \\
\hline Hydroxyurea $^{\circ}$ & - & $10(17.5)$ & - & - \\
\hline Chronic blood transfusions $^{\circ}$ & - & $13(22.8)$ & - & - \\
\hline $\mathrm{HSCT}^{\circ}$ & - & $2(3.5)$ & - & - \\
\hline
\end{tabular}

*Were excluded patients with unknown immunization status $(\mathrm{n}=17)$ or patients not eligible $(\mathrm{n}=14)$ i.e children born before 2000 (date of licensing of PCV7) or who died before 2 months of age; **Were excluded patients with unknown immunization status $(n=7)$ or patients not eligible ( $n=23$ ) i.e children who died before 24 months $* * *$ Were excluded patients with missing data $(\mathrm{n}=10)^{\circ}$ More than one disease modifying therapy was possible. \% is calculated out of total patients with available data ; PV23: polysaccharidic vaccine 23; PCV 7 or 13: pneumococcal conjugate vaccine 7 or 13; HSCT: hematopoietic stem cell transplantation 
formed based on the number of fatal cases of children with SCD born during the study period over the number of PY of follow-up of the cohort diagnosed at birth during the study period (open cohort). Children born after January 1, 2000 contributed to PY from diagnosis of SCD at birth to either death or December 31, 2015, whichever came first. To ensure exhaustivity, the National Death Certificates Registry was also interrogated. This study was approved by the French authorities in accordance with the French MR004 methodology $\left(n^{\circ} 20181109114235\right)$.

During the study period, 4,682 babies were born and diagnosed with SCD in mainland France following the targeted NBS program, contributing to 35,291 PY of follow-up until December 31, 2015. During the same period, 84 deaths of children with SCD matching our inclusion criteria were reported by all but one center of the SCD network (49 centers, $98 \%$ response rate) (Figure 1). Cross examination of the National Death Certificates Registry yielded a comparable figure of 81 deaths. Of the
84 reported cases, 9 were excluded because main followup was performed abroad or due to significant missing data. A total of 75 cases of death were thereafter analyzed in detail, including 57 children born and dead between 2000 and 2015 who contributed to the estimation of the death incidence rate in the cohort.

Death incidence rate in the open cohort was 57 of 35,291 (0.16/ 100 PY) for all causes but dropped to 31 of 35,436 (0.09/ 100 PY) when considering SCD-related causes only. In children under 5 years old, however, the death incidence rate of 41 of 4,733 was higher than the whole cohort for all causes (0.87/ 100 PY) and for SCDrelated causes with 25 of 4,837 (0.52/ 100 PY).

Main characteristics of the patients, death classification in relation to SCD and place of death are summarized in Table 1. Of note, no case of death occurred following a failure of the targeted NBS program. Among causes of death unrelated to SCD $(n=18)$, other serious medical conditions caused the fatal outcome in 13 cases $(72.2 \%)$ : congenital disorder $(n=7)$, premature birth $(n=3)$, neona-
Fatal cases reported by the French National SCD

Network with the following criteria:

$S C D$ diagnosis

Fatal outcome between 01/01/2000 and $31 / 12 / 2015$

$<18$ years at time of death
Fatal cases reported by the French National Death

Registry* with the following criteria:

- SCD as main or associated cause of death (ICD 10 thesaurus: D57)

- Fatal outcome between $01 / 01 / 2000$ and 31/12/2015 in metropolitan France

$<18$ years at time of death
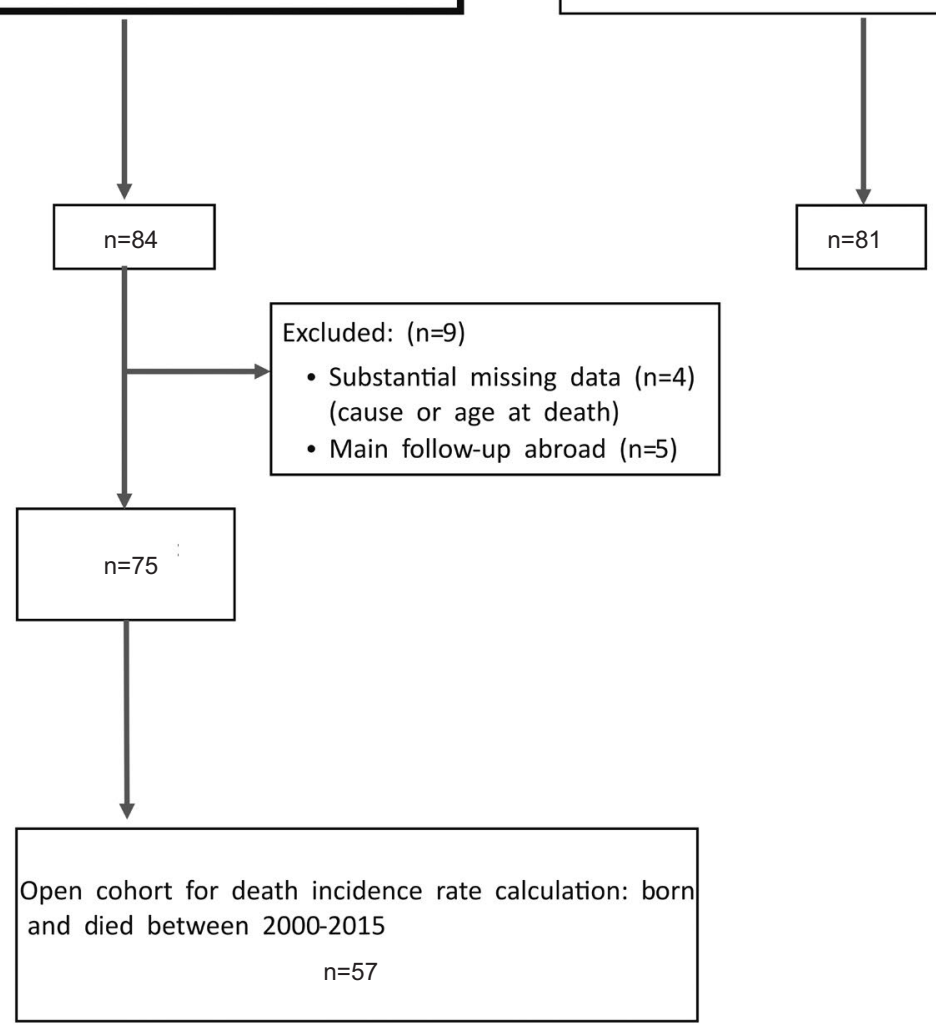

Figure 1. Flow chart of study. A total of 75 of 84 reported cases were analyzed in detail, of which 52 children were born and dead between January 1,2000 and December 31, 2015 who constituted the open cohort for death incidence rate calculation. The National Death Registry reported during the same period 81 pediatric death certificates with sickle cell disease as principal or associated cause of death. 
Table 2. Causes of sickle cell disease-related death.

\begin{tabular}{|c|c|c|c|}
\hline SCD-related causes, n \%* & $\begin{array}{l}\text { SCD (All genotypes) } \\
53 \text { (100) }\end{array}$ & $\begin{array}{c}\text { SS } / \text { S }^{0} \text { thalassemia/S-D Punjab } \\
49(92.5)\end{array}$ & $\begin{array}{c}S C / S \beta^{+} \text {thalassemia } \\
4(7.5)\end{array}$ \\
\hline Infection, n (\%)* & $21(39.6)$ & $20(95.2)$ & $1(4.8)$ \\
\hline Median age at death, Years (range) & $3.5(1.2-14.9)$ & $3.4(1.2-14.9)$ & 11 \\
\hline Pneumococcal invasive infection & $8(15.1)$ & $8(15.1)$ & - \\
\hline Hematological, n (\%)* & $15(28.3)$ & $13(24.5)$ & $2(3.8) * *$ \\
\hline Median age at death, Years (range) & $2.87(0.2-15.3)$ & $3.8(0.2-15.3)$ & $1.7(1.5-1.9)$ \\
\hline Acute splenic sequestration & $9(17)$ & 7 & $2 * *$ \\
\hline Other acute anemia & $6(11.3)$ & $6(11.3)$ & - \\
\hline Neurovascular, n (\%)* & $8(15.1)$ & $8(15.1)$ & - \\
\hline Median age at death, Years (range) & $13.6(4.7-17)$ & $13.6(4.7-17)$ & - \\
\hline Stroke & $3(5.7)$ & 3 & - \\
\hline Cerebral hemorrhage & $3(5.7)$ & 3 & - \\
\hline Miscellaneous & $2(3.8)$ & 2 & - \\
\hline Cardiothoracic, n (\%)* & $7(13.2)$ & $6(11.3)$ & $1(1.9)$ \\
\hline Median age at death, Years (range) & $6.75(3.17-17.9)$ & $6.71(3.17-17.9)$ & 13.5 \\
\hline Acute chest syndrome & $6(11.3)$ & 6 & - \\
\hline Pulmonary embolism & $1(1.9)$ & - & 1 \\
\hline Other: Post HSCT***, n (\%)* & $2(3.8)$ & 2 & - \\
\hline Median age at death, Years (range) & $12.7(10-15.4)$ & $12.7(10-15.4)$ & - \\
\hline
\end{tabular}

tal infection $(n=3)$; and was accidental in the remaining $(n=5)$. Regarding deaths abroad $(n=4)$, all occurred in homozygous children aged less than 2 years while travelling in sub-Saharan Africa, of which three were secondary to acute anemia and one was of unknown cause.

SCD-related causes of death $(n=53)$ are detailed in Table 2. In this category, the majority of patients had an accordingly severe genotype (SS/S $\beta^{0}$-thalassemia/ $\mathrm{S}$-DPunjab). Infection was the overall leading cause of death $(n=21)$, with a frequent presentation of septic shock ( $n=13 ; 61.9 \%)$. A microbiological cause was identified in $11(52.3 \%)$ cases: 9 bacterial infections (8 $S$. pneumoniae, one E. Coli), 1 dengue and 1 malaria. In 4 of 8 cases of pneumococcal infection, the serotypes were determined (12F, 15B, 23A and 24F) and, for 2 of them, were included in the polysaccharidic vaccine $(12 \mathrm{~F}$ and 15B). In those two cases, although children had been appropriately vaccinated by the polysaccharidic vaccine, they were reported to be non-compliant with penicillin prophylaxis. There were no deaths from meningococcal or Haemophilus influenzae type $\mathrm{b}$ infection. Of note, among anemia-related deaths $(n=15), 6$ were associated with an infectious event, including 2 cases of malaria. Fatal neurological and cardiopulmonary complications expectedly occurred in older children $(n=15)$. Likewise, age at death occurred later in patients who received disease-modifying therapies $(n=18$, median age 10 years, range: 3-18), and neuro-vascular complications $(n=7$; $46.7 \%)$ and infections $(n=3 ; 20 \%)$ were the main related causes.

Analysis of time to therapeutic care was available in 44 of $53(83 \%)$ cases regarding SCD-related deaths. A significant delay was reported in 9 of them $(20.4 \%)$ and resulted from an inappropriate medical evaluation in 4 cases. Out of $47(88.6 \%)$ medical files in which compliance data was reported, a significant concern was notified in 13 cases $(27.6 \%)$, all in children less than 7 years old. Of them $10(76.9 \%)$ had a SS/S $\beta^{0}$-thalassemia genotype and died from an acute infectious condition. Regarding access to medical care, in 34 of 44 patients $(77.1 \%)$ with available data, access to the nearest hospital took theoretically over 30 minutes by car. We were however unable to test whether this finding significantly influenced the fatal outcome. Altogether, among SCDrelated causes of deaths, a total of 18 cases $(33.9 \%)$ were negatively impacted by either a delayed medical intervention and/or a significant lack of adherence to medical advice.

This nationwide study shows that currently in mainland France, all-causes death incidence rate in children with SCD is globally low (0.16/ 100 PY), and so is disease-related deaths. Findings align with death rates reported in expert single center cohorts in high-income countries (0.13-0.2/ $100 \mathrm{PY}) .{ }^{1,8,9}$ However, in children under five, the death incidence rate was not only higher than the whole cohort but also mainly a direct consequence of the disease (0.87/100 PY and 0.52/ $100 \mathrm{PY}$ for all causes and SCD-related causes, respectively). Contrary to studies based on death certificate analysis, our methodology allowed for precise causes of death to be detailed and showed that the latter were mainly due to preventable acute complications, namely infection and acute anemia.

Infection was the leading cause and was furthermore presumably underestimated given that half of deaths attributed to acute anemia also occurred in a context of infection. Pneumococcal invasive infection per se represented $15,1 \%$ of SCD-related causes in this study further underlying the ongoing pneumococcal burden in younger children. ${ }^{10,11}$ Acute splenic sequestration was the second cause of death, a distressing finding given that these children were diagnosed with SCD and urgent blood transfusion is a readily available life-saving treatment. Of note, two of these children had a S- $\beta^{+}$-thalassemia geno- 
type, less commonly associated with this complication, although the severity of the mutation was unknown. Another salient finding is the proportion of SCD-related deaths $(33 \%)$ which could perhaps have had a different outcome with improved adherence to antibiotic prophylaxis and/or vaccination coverage and/or faster medical intervention, highlighting the potential for specific improvement.

This study, by its design and methodology has several limitations. Defining the role of SCD in the death sequence was a thorny issue, particularly in case of incomplete data, and may have led to a fraction of misclassified causes of death. Another limitation relates to the calculation of death incidence rates. As there is no national SCD registry in France, our methodology was based on the extrapolation of the cohort screened at birth, thereby excluding children with SCD born abroad who arrived in France during the study period. Notwithstanding, comparison with the national death registry confirmed near exhaustivity in the numbers of reported deaths.

Altogether, these results show that children with SCD are at increased risk of death by specific acute complications. They highlight the ongoing vulnerability of younger children with SCD despite current pneumococcal prophylactic measures and easy access to care in France. SCD requires a high level of parental involvement, education, adherence and attention to symptoms. Improved empowerment of families through better communication targeting pneumococcal prophylaxis and spleen palpation in particular, close monitoring of younger patients by dedicated staff and increased utilization of disease-modifying treatment are likely to further decrease mortality rates in the future.

Emilie Desselas, ${ }_{1}^{1}$ Isabelle Thuret, ${ }^{2}$ Florentia Kaguelidou, ${ }^{3,4}$ Malika Benkerrou, Mariane de Montalembert, ${ }^{1,6}$

Marie-Hélène Odièvre, Emmanuelle Lesprit, ${ }^{7}$ Eva Rumpler, Arnaud Fontanet, ${ }^{9,10}$ Corinne Pondarre ${ }^{11,12}$

and Valentine Brousse ${ }^{1,6,13}$

${ }^{1}$ Service de Pédiatrie Générale et Maladies Infectieuses, Centre de Référence de la Drépanocytose, Hôpital Necker-Enfants Malade, Paris; ${ }^{2}$ Service d'Hématologie pédiatrique, CHU La Timone, Marseille; ${ }^{3}$ Centre d'Investigations Cliniques, INSERM CIC1426, Hôpital Robert Debré, APHP, Paris; ${ }^{4}$ Université Paris Diderot, UMR 123, ECEVE, Paris; ${ }^{5}$ Service de Pédiatrie Générale, Centre de Référence de la Drépanocytose, Hôpital Robert Debré, Paris; ${ }^{6} G R-E X$, Paris; ${ }^{7}$ Service de Pédiatrie Générale, Hôpital Louis Mourier, Colombes; ${ }^{8}$ Service de Pédiatrie Générale, Centre de Référence de la Drépanocytose, Hôpital Trousseau, Paris; ${ }^{9}$ Epidémiologie des Maladies Emergentes, Département de Santé Globale, Institut Pasteur, Paris;
${ }^{10}$ Unité PACRI, Conservatoire National des Arts et Métiers, Paris; ${ }^{1}$ Service de Pédiatrie Générale, Centre de Référence de la

Drépanocytose, Centre Intercommunal de Créteil, Créteil; ${ }^{12}$ Inserm

U955, Université Paris XII, Créteil and ${ }^{13}$ Inserm U1134,

Université de Paris, Paris, France

Correspondence:

VALENTINE BROUSSE - valentine.brousse@gmail.com

\section{doi:10.3324/haematol.2019.237602}

Acknowledgments: we wish to thank all the investigators of this study in particular all the clinicians in care of patients who reported these cases. We wish to thank Mireille EB (Inserm - CépiDc, C.H.U. de Bicêtre). We are indebted to Emmanuelle Varon from the Centre National de Référence des Pneumocoques, Centre Intercommunal de Créteil, for providing pneumococcal serotypes. We are also very thankful to the National French network: Filiere Nationale des Maladies Rares du Globules Rouge et de l'Erythropoiese for their logistic help and the daily support in the care of patients with sickle cell disease.

\section{References}

1. Telfer P, Coen $\mathrm{P}$, Chakravorty S, et al. Clinical outcomes in children with sickle cell disease living in England: a neonatal cohort in East London. Haematologica. 2007;92(7):905-912.

2. Gill FM, Sleeper LA, Weiner SJ, et al. Clinical events in the first decade in a cohort of infants with sickle cell disease. Cooperative Study of Sickle Cell Disease. Blood. 1995;86(2):776-783.

3. Yanni E, Grosse SD, Yang Q, Olney RS. Trends in pediatric sickle cell disease-related mortality in the United States, 1983-2002. J Pediatr. 2009;154(4):541-545

4. Kremp O, Paty AC, Suzan F, et al. [Mortality due to sickle cell anaemia in France from birth to 18 years of age]. Arch Pediatr. 2008;15(5):629-632

5. Hamideh D, Alvarez $\mathrm{O}$. Sickle cell disease related mortality in the United States (1999-2009). Pediatr Blood Cancer. 2013;60(9):14821486

6. Chaturvedi S, DeBaun MR. Evolution of sickle cell disease from a life-threatening disease of children to a chronic disease of adults: The last 40 years. Am J Hematol. 2016;91(1):5-14

7. Prise en charge de la drépanocytose chez l'enfant et l'adolescent. Haute Autorité de Santé. https://www.has-sante.fr/jcms/ c_272479/fr/prise-en-charge-de-la-drepanocytose-chez-l-enfant-et-1adolescent.

8. Wang Y, Liu G, Caggana M, et al. Mortality of New York children with sickle cell disease identified through newborn screening. Genet Med. 2015;17(6):452-459.

9. Quinn CT, Rogers ZR, McCavit TL, Buchanan GR. Improved survival of children and adolescents with sickle cell disease. Blood. 2010; 115(17):3447-3452.

10. Oligbu G, Fallaha M, Pay L, Ladhani S. Risk of invasive pneumococcal disease in children with sickle cell disease in the era of conjugate vaccines: a systematic review of the literature. Br J Haematol. 2019;185(4):743-751.

11. Streetly A, Sisodia R, Dick M, Latinovic R, Hounsell K, Dormandy E. Evaluation of newborn sickle cell screening programme in England: 2010-2016. Arch Dis Child. 2018;103(7):648-653. 